\title{
Diagnosis, clinicopathological characteristics and prognosis of pulmonary mucinous adenocarcinoma
}

\author{
FEI SUN ${ }^{1}$, PENGCHENG WANG $^{1}$, YIMING ZHENG ${ }^{1}$, WEIGUANG JIA ${ }^{1}$, \\ FUXING LIU $^{2}$, WEI XIAO ${ }^{2}$, JINGJING BAO ${ }^{2}$, SONG WANG ${ }^{1}$ and KAIJIN LU ${ }^{1}$ \\ Departments of ${ }^{1}$ Cardiothoracic Surgery and ${ }^{2}$ Pathology, Taizhou People's Hospital, Taizhou, Jiangsu 225300, P.R. China
}

Received September 19, 2016; Accepted September 15, 2017

DOI: $10.3892 / \mathrm{ol} .2017 .7312$

\begin{abstract}
Primary pulmonary mucinous adenocarcinoma (PPMA) is an uncommon subtype of lung adenocarcinoma. The present study attempted to clarify the diagnosis, clinicopathological characteristics, and pathologic significance of epithelial growth factor receptor (EGFR) and Kirsten rat sarcoma viral oncogene (KRAS) mutations and the prognosis of PPMA. A total of 29 patients with PPMA from among 1,469 surgically resected patients with lung adenocarcinoma were enrolled. All of the tumours expressed CK7 and 5 cases exhibited co-expression with CK20. A total of 8 cases expressed EGFR, 14 cases expressed P53 and 2 cases expressed CEA. The majority of mucinous adenocarcinomas expressed thyroid transcription factor 1, Napsin A, Villin and Cam5.2 proteins. KRAS mutations were observed in $62 \%$ of patients and were more prevalent in the lower lung lobe and in patients with invasive mucinous adenocarcinoma. A total of 2 cases exhibited an EGFR mutation, and the co-mutation of KRAS and EGFR was only detected in 1 case. The relapse-free and overall survival rates at 5 years were 70.4 , and $81.5 \%$, respectively. The results may assist to identify a molecular target and supply important information for a therapeutic strategy for patients with PPMA.
\end{abstract}

\section{Introduction}

Lung carcinoma is the most common type of cancer and is the leading cause of cancer-associated mortality in humans worldwide; non-small-cell lung cancer (NSCLC) accounts for $80 \%$ of all lung cancer cases (1). Adenocarcinoma is the most common pathological subtype of NSCLC and has demonstrated an increasing prevalence (2). Primary pulmonary mucinous adenocarcinoma (PPMA) includes a group of subtypes of mucin-producing adenocarcinomas in the lung, and was first

Correspondence to: Professor Pengcheng Wang, Department of Cardiothoracic Surgery, Taizhou People's Hospital, 210 Yingchun West Road, Taizhou, Jiangsu 225300, P.R. China

E-mail: pengch_wang@163.com

Key words: mucinous adenocarcinoma, Kirsten rat sarcoma viral oncogene, epithelial growth factor receptor identified in 1978 as a mucous cyst tumour of the lung (3); other terms including cystic mucinous adenocarcinoma, colloid adenocarcinoma and mucinous bronchioloalveolar carcinoma were subsequently described (4,5). A novel classification for lung adenocarcinoma was proposed by an international multidisciplinary expert panel from World Health Organization (WHO) in 2015 (6) and invasive mucinous adenocarcinoma (IMA) was listed as an independent variant subtype. The new classification system of the concept of mucinous adenocarcinoma has been devised and is generally accepted. Referring to all of the subtypes in the lung with the mucin-producing phenotype, PPMA includes IMA, mucinous minimally invasive adenocarcinoma (mMIA) and mucinous adenocarcinoma in situ (mAIS). Compared with other lung adenocarcinoma subtypes, IMA exhibits different immunohistochemical and molecular features, and a different prognosis $(7,8)$.

A total of $5-10 \%$ of lung adenocarcinoma cases exhibit features of mucinous differentiation (9), which provide specific challenges for pathological diagnosis. PPMA may be indistinguishable from metastatic neoplasms of gastrointestinal origin in terms of histology, and lung mucinous adenocarcinomas are different from non-mucinous adenocarcinomasin terms of immunohistochemical profiles, including the expression of cytokeratin 20 (CK20), caudal type homeobox 2 (CDX2), and hepatocyte nuclear factor $4 \alpha$ and the lack of expression of thyroid transcription factor 1 (TTF-1) and Napsin A (9).

The accumulation of genetic abnormalities is considered to induce the development of malignant neoplasms. Kirsten rat sarcoma viral oncogene (KRAS) and epidermal growth factor receptor (EGFR) mutations are frequently detected in patients with lung cancer $(10,11)$. The KRAS mutation is the most common driver mutation in human cancer, although the prevalence of mutations and the affected codons differ according to the type of cancer. The majority of the available studies that included all types of adenocarcinoma and employed low sensitive and single-gene technologies have established that KRAS mutations occur in $61 \%$ of IMAs, and are associated with poor prognosis and a lack of response to chemotherapy (12). In addition, the EGFR mutation has been an area of particular interest on account of the identification that the application of EGFR-tyrosine kinase inhibitors in clinical tests resulted in a rapid response among patients with pulmonary adenocarcinoma who carried a mutation in EGFR (1). Furthermore, mutations in EGFR and KRAS are usually mutually exclusive (13). 
The purpose of the present study was to clarify the clinicopathological characteristics, KRAS and EGFR mutation status, and prognosis of patients with PPMA. The results may assist to identify a molecular target and supply important data for therapeutic strategy for patients with PPMA.

\section{Materials and methods}

Patient selection. Between January 2001 and May 2011, a total of 1,469 patients with lung adenocarcinoma were surgically treated in the Department of Cardiothoracic Surgery, Taizhou People's Hospital (Taizhou, China). From these, 29 patients (2\%) with PPMA were selected. Inclusion criteria were as follows: i) the presence of a mucinous pattern; ii) the lack of a prior non-lung related cancer diagnosis; iii) the presence of accurate clinical follow-up data, as well as the availability of residual tissue for genetic analyses. The study was reviewed and approved by the institutional review board of Taizhou People's Hospital (authorization number: KY201600301). Due to the retrospective nature of the present study, the requirement for written informed consent was waived.

Clinical information. All available clinical information was collected for analyses from the medical records and reports that were completed by the referring physicians. Sex, age at diagnosis, smoking status, tumour size, stage and location were included.

Histological classification. The surgically resected specimens from each patient were fixed for $24 \mathrm{~h}$ in $10 \%$ neutral buffered formalin solution in phosphate buffered saline $(\mathrm{pH} 7.4)$ at room temperature. Following fixation, the samples were washed with distilled water, dehydrated in graded alcohol and embedded in paraffin and sectioned at a thickness of $4 \mathrm{~mm}$. Histological slides were reviewed and sections were stained with hematoxylin and eosin (H\&E; Beijing Leagene Biotech Co., Ltd., Beijing, China) according to the manufacturer's protocol at room temperature, and all available H\&E-stained sections were examined by two researchers (Dr Liu FX and Dr Bao JJ, Department of Pathology, Taizhou People's Hospital, Jiangsu, China) in a blinded fashion under an optical microscope (Olympus BX41; Olympus Corporation, Tokyo, Japan). Classification was performed according to the International Association for the Study of Lung Cancer recommendations (14). Invasive mucinous adenocarcinoma was defined by goblet or columnar cell morphology with abundant intracytoplasmic mucin present in $>90 \%$ of tumour cells. Mixed mucinous/nonmucinous adenocarcinoma was defined by the presence of mucinous and nonmucinous components in $\geq 10 \%$ of tumour cells.

Immunohistochemical analysis. Immunohistochemical analysis for markers of pulmonary differentiation was performed on one slide of tumour tissue per case. The primary antibodies that were used were as follows: TTF-1 (1:200 dilution; cat. no. IR056; Dako; Agilent Technologies, Inc., Santa Clara, CA, USA), Napsin A (1:200 dilution; cat. no. NCL-L-Napsin A; Leica Microsystems, Inc., Buffalo Grove, IL, USA), cytokeratin 7 (CK7; 1:200 dilution; cat. no. IR619; Dako; Agilent Technologies, Inc.), CK20 (1:200 dilution; cat. no. IR777;
Dako; Agilent Technologies, Inc.), EGFR (1:100 dilution; cat. no. NCL-L-EGFR; Leica Microsystems, Inc.), tumor protein (P)53 (1:200 dilution; cat. no. IR616; Dako; Agilent Technologies, Inc.), carcinoembryonic antigen (CEA; 1:200 dilution; cat. no. IR622; Dako; Agilent Technologies, Inc.), CAm5.2 (1:100 dilution; cat. no. RTV-CAM5.2-0.11; Leica Microsystems, Inc.) and Villin (1:200 dilution; cat. no. IR076; Dako; Agilent Technologies, Inc.) antibodies. Staining of $<5 \%$ of tumour cells was interpreted as negative, staining of $5-80 \%$ of tumour cells was classified as mixed, and staining in $>80 \%$ of tumour cells was interpreted as positive. Histological classification and the interpretation of the immunohistochemical results were based on a consensus review by three pathologists.

EGFR and KRAS mutation analysis. The tumor tissues were surgically resected for all patients and then fixed for $24 \mathrm{~h}$ in $10 \%$ neutral buffered formalin solution at room temperature, washed in distilled water, dehydrated in graded alcohol, embedded in paraffin. Tumor tissues were dissected from unstained histological sections. DNA from the tumor tissues was isolated and purified using the DNA Tissue Isolation kit (Qiagen GmbH, Hilden, Germany) according to the manufacturer's protocol. KRAS and EGFR mutations were subsequently identified using the Human KRAS and EGFR Mutation Detection kit (Amoy Diagnostics, Xiamen, China) according to the manufacturer's protocol. Mutational analyses of EGFR gene exons 18, 19, and 21 and KRAS exons 12 and 13 were performed using the loop-hybrid mobility shift assay and sequencing as described previously (15).

Statistical analysis. All analyses were performed using SPSS 19.0 software (IBM Corp., Armonk, NY, USA). Data are expressed as the mean \pm standard deviation. The $\chi^{2}$ test and Fisher's exact test were used to compare the observed percentages. Fisher's exact test was performed if there were $\leq 5$ observations in a group. $\mathrm{P}<0.05$ was considered to indicate a statistically significant difference.

\section{Results}

Clinicopathological characteristics. Of the 1,469 lung adenocarcinoma tumours, 29 tumours were PPMA. The clinicopathological data for these cases are summarised in Table I. Of these patients, 12 were male and 17 were female, and the median age was 69 years (ranging from 35 to 83 years old). Smokers (smoking index $\geq 200$; smoking index equalled the average root number of cigarettes smoked per day multiplied by number years of smoking) accounted for $34.5 \%$ (10/29) of the patients. The maximum diameters of the tumours ranged from $0.9-13 \mathrm{~cm}$. The predominant histologic patterns were IMA (72.4\%, 21/29), AIS and MIA $(27.6 \%, 8 / 29)$. A total of $31 \%$ $(9 / 29)$ of patients presented with pleural invasion. Lymphatic invasion was observed in 10 cases (34.5\%). No instances of pulmonary metastasis or vascular invasion were observed.

Immunohistochemistry analysis. Nuclear staining was observed for TTF-1 and P53, and cytoplasmic staining was observed for Napsin A, CK7, CK20, EGFR, CEA, CAm5.2 and Villin. Staining indicated that TTF1 expression was elevated 
Table I. Clinicopathological parameters of the 29 patients with mucinous adenocarcinoma included in the present study.

\begin{tabular}{|c|c|}
\hline Parameter & $\mathrm{n}(\%)$ \\
\hline \multicolumn{2}{|l|}{ Age (years) } \\
\hline$<65$ & $13(44.8)$ \\
\hline$\geq 65$ & $16(55.2)$ \\
\hline \multicolumn{2}{|l|}{ Sex } \\
\hline Male & $12(41.4)$ \\
\hline Female & $17(58.6)$ \\
\hline \multicolumn{2}{|l|}{ Smoking index } \\
\hline$<200$ & $19(65.5)$ \\
\hline$\geq 200$ & $10(34.5)$ \\
\hline \multicolumn{2}{|c|}{ Tumour size (cm) } \\
\hline$\leq 3$ & $18(62.1)$ \\
\hline$>3$ & $11(37.9)$ \\
\hline \multicolumn{2}{|l|}{ Lobe } \\
\hline Upper/middle & $6(20.7)$ \\
\hline Lower & $23(79.3)$ \\
\hline \multicolumn{2}{|l|}{ Stage } \\
\hline I-II & $20(69)$ \\
\hline III-IV & $9(31)$ \\
\hline \multicolumn{2}{|c|}{ Histological subtype } \\
\hline AIS and MIA & $8(27.6)$ \\
\hline IMA & $21(72.4)$ \\
\hline \multicolumn{2}{|l|}{ Pleural invasion } \\
\hline Positive & $3(10.3)$ \\
\hline Negative & $26(89.7)$ \\
\hline \multicolumn{2}{|c|}{ Lymphatic permeation } \\
\hline Positive & $10(34.5)$ \\
\hline Negative & $19(65.5)$ \\
\hline \multicolumn{2}{|c|}{ Vascular invasion } \\
\hline Positive & $0(0)$ \\
\hline Negative & $29(100)$ \\
\hline
\end{tabular}

AIS, adenocarcinoma in situ; MIA, minimally invasive adenocarcinoma; IMA, invasive mucinous adenocarcinoma.

in 18/29 (62.1\%) cases. Positive CK7 expression was identified in all cases; $100 \%$ of tumour cells expressed this cytokeratin, but $5(17.2 \%)$ tumours co-expressed CK20. A total of $8(27.6 \%)$ cases expressed EGFR, 14 (48.3\%) cases expressed P53, but only $2(6.9 \%)$ cases expressed CEA. There were $9(31 \%)$ cases that expressed Napsin A. In all of the cases, a large proportion of carcinoma cells expressed Villin and 11 (37.9\%) cases expressed Cam5.2. The co-expression of CDX2 and TTF1 was not identified within single tumour cells (Fig. 1).

EGFR and KRAS mutations. The EGFR and KRAS mutation statuses of the patients are summarised in Table II. A total of 18 cases $(62 \%)$ exhibited a KRAS mutation and an EGFR mutation was identified in 2 cases (6.9\%). Exon 12 was mutated in all of the KRAS mutation cases, while the 2 EGFR
Table II. KRAS and EGFR status of 29 patients with mucinous adenocarcinomas.

\begin{tabular}{lc}
\hline Mutation & $\mathrm{n}(\%)$ \\
\hline EGFR & \\
Exons 18 & 0 \\
Exons 19 & 0 \\
Exons $21(\mathrm{~L} 858 \mathrm{R})$ & $2(6.9)$ \\
None & $27(93.1)$ \\
KRAS & \\
Exons 12 & $18(62)$ \\
Exons 13 & 0 \\
None & $11(38)$
\end{tabular}

EGFR, epithelial growth factor receptor; KRAS, Kirsten rat sarcoma viral oncogene.

mutations occurred in exon 21 (L858R) of EGFR. A KRAS and EGFR co-mutation was detected in 1 case. A total of 10 patients exhibited neither EGFR nor KRAS mutations.

Association between the clinicopathological characteristics and KRAS and EGFR mutations. In the 29 patients with PPMA, KRAS mutations were significantly more likely to be located in the lower lung lobe $(73.9 \%, 17 / 23)$ compared with in the upper/middle lobe $(16.7 \%, 1 / 6 ; \mathrm{P}=0.036)$. Additionally, the number of tumours with a KRAS mutation was increased in patients with invasive mucinous adenocarcinoma $(76.2 \%$, $16 / 21)$ compared with in other subtypes (25\%, 2/8; $\mathrm{P}=0.035)$. No significant associations were identified between KRAS or EGFR mutations and other clinicopathological parameters (Table III). There were no significant associations between histological features, including lymphatic permeation or pleural invasion, and KRAS or EGFR mutations (Table III).

Prognosis following resection. The clinical outcomes of patients with Mas were then examined. The median follow-up period for the surviving patients was 74.8 months (range, 34-132 months). No serious complications following the surgeries and no surgical mortalities were noted. A total of 1 patient was lost to follow-up, and none of the 3 patients with AIS or the 5 patients with MIA exhibited recurrences. A total of 1 patient with IMA succumbed to other diseases with no evidence of recurrence. A total of 8 patients exhibited recurrences. With regard to the site, the first recurrence was an ipsilateral pulmonary metastasis in 3 patients and bilateral pulmonary metastasis in 5 patients. Of these patients, 5 succumbed to recurrent lung cancer and 3 were alive with disease. The 11 patients with IMA were asymptomatic, and are currently being managed with close radiologic and clinical follow-up. The overall and relapse-free survival rates at 5 years were 70.4 , and $81.5 \%$, respectively.

\section{Discussion}

The 2015 WHO Classification of Lung Tumors: Impact of Genetic, Clinical and Radiologic Advances Since the 2004 

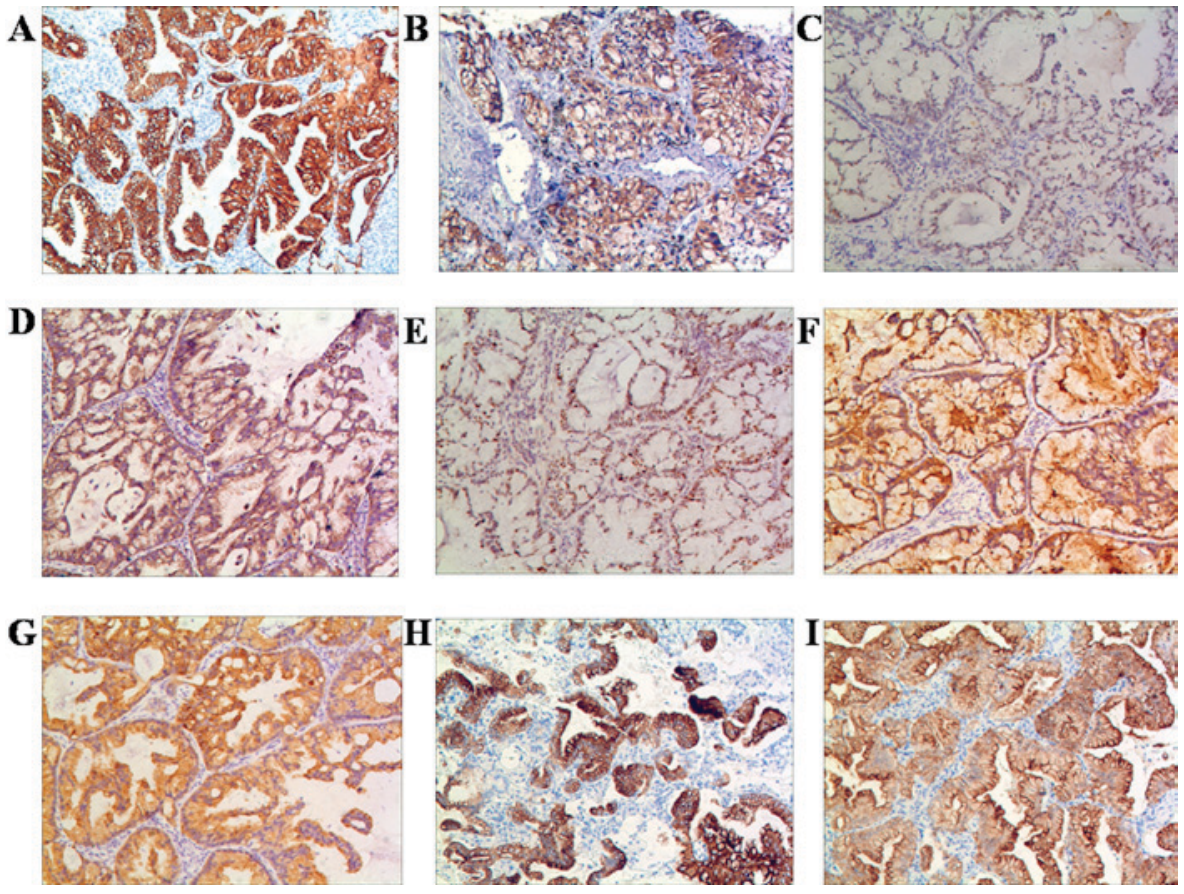

Figure 1. The results of immunohistochemical analysis of the resected nodules from 29 patients, the tumour cells exhibited (A) strong diffuse cytoplasmic immunoreactivity for CK7 (29/29; 100\%); (B) intense cytoplasmic staining for EGFR (8/29; 27.6\%); (C) nuclear staining for TTF-1 (18/29, 62.1\%); (D) intense cytoplasmic staining for Napsin A $(9 / 29 ; 31 \%)$; (E) nuclear staining for P53 (14/29; 48.3\%); (F) moderate cytoplasmic staining for CEA (2/29; 6.9\%); (G) moderate cytoplasmic staining for Cam5.2 (11/29; 37.9\%); (H) cytoplasmic staining for CK20 (5/29; 17.2\%); and (I) intense cytoplasmic staining for Villin $(15 / 29 ; 51.7 \%)$. Scale bar, $50 \mu \mathrm{m}$. CK, cytokeratin; EGFR, epithelial growth factor receptor; TTF-1, thyroid transcription factor 1; CEA, carcinoembryonic antigen; p53, tumour protein 53 .

Classification has been published recently (6). Compared with the 2004 WHO Classification, there are multiple major changes for common types of lung cancer, the majority of which follow the 2011 lung adenocarcinoma classification system that was sponsored by the International Association for the Study of Lung Cancer (IASLC)/American Thoracic Society/European Respiratory Society, which has essentially been adopted with minor changes (14). IMAs, which account for 2-10\% of lung adenocarcinoma cases in East Asia, Europe, and the United States (16-18), were considered to demonstrate increased levels of malignancy compared with other common subtypes of lung adenocarcinoma, including lepidic and acinar subtypes (19).

Lung adenocarcinoma has been associated with poor recurrence-free survival and adverse prognosis, as the disease often presents with minimal or misleading clinical symptoms, and multifocal heterogeneous unresectable lesions (20). In the present study, PPMA was classified as AIS, MIA or IMA according to the new classification of lung adenocarcinoma by IASLC (14). The diagnosis of AIS, MIA or IMA cannot be made without the complete histological evaluation of the entire tumour. In the present study, the maximum diameters of the tumours ranged from $0.9-13 \mathrm{~cm}$. Pleural invasion was observed in 9 cases (31\%) and lymphatic invasion was present in 10 cases $(34.5 \%)$.

In the present study, CK7 expression was identified in all of the PPMA tumours, most often combined with TTF1, EGFR, P53, Napsin A, Villin or Cam5.2 expression. Only a minority of tumours expressed CK20 and CEA. Geles et al (21) confirmed that the number of TTF1-negative mucinous adenocarcinomas was quite high, which may suggest differences between a Caucasian and an Asian population. In the study by Kunii et al (22), MUC2 and MUC5AC expression, together with $\mathrm{HNF} 4 \alpha$, was observed in cases that were negative for TTF1.

The presence of EGFR mutations has been demonstrated as a predictive factor for a goodresponse to EGFR-tyrosine kinase inhibitors $(15,23)$. Mucinous adenocarcinoma is associated with the absence of EGFR mutations and the presence of KRAS mutations (24). One previous study (25) indicated that EGFR mutations are not commonly detected in patients with mucinous adenocarcinoma. In the present study, it was indicated that invasive mucinous adenocarcinoma of the lung is genetically and clinically distinct. EGFR mutations were detected in $2 / 29$ patients $(6.9 \%)$, and these mutations have been previously described to range from 0 to $22 \%$ (25). KRAS mutations were detected in $18 / 29$ patients (62\%) in the present study, in concordance with the data of previous studies in which these mutations have been previously described to range from 42.5 to $86 \%(7,23,25)$. The results of the present study for EGFR and KRAS mutations were similar to those in previous studies. The KRAS mutations may be associated with the carcinogenesis of mucinous adenocarcinoma. In terms of specific KRAS mutations, exon 12 was most common in the cohort of the present study. KRAS exon 12 is most common in colorectal and pancreatobiliary carcinoma, suggesting that IMA of the lung may have more in common with pancreatobiliary, and intestinal tract cancer types (26).

Several studies have analysed the association between KRAS or EGFR mutations and the clinicopathological characteristics in pulmonary carcinoma. The results of the present study suggest that KRAS or EGFR mutations in 
Table III. Clinicopathological parameters of the 29 patients with primary pulmonary mucinous adenocarcinoma included in the present study.

\begin{tabular}{|c|c|c|c|c|c|c|}
\hline \multirow{3}{*}{$\begin{array}{l}\text { Clinicopathological } \\
\text { parameters }\end{array}$} & \multicolumn{5}{|c|}{ Mutation } & \multirow[b]{3}{*}{ P-value } \\
\hline & \multicolumn{2}{|c|}{ KRAS } & \multirow[b]{2}{*}{$\mathrm{P}$-value } & \multicolumn{2}{|c|}{ EGFR } & \\
\hline & Positive $(n=18)$ & Negative $(n=11)$ & & Positive $(n=2)$ & Negative $(n=27)$ & \\
\hline Age (years) & & & 0.271 & & & 1 \\
\hline$<65$ & 10 & 3 & & 1 & 12 & \\
\hline$\geq 65$ & 8 & 8 & & 1 & 15 & \\
\hline $\operatorname{Sex}$ & & & 0.461 & & & 1 \\
\hline Male & 6 & 6 & & 0 & 12 & \\
\hline Female & 12 & 5 & & 2 & 15 & \\
\hline Smoking index & & & 0.298 & & & 0.532 \\
\hline$<200$ & 10 & 9 & & 1 & 19 & \\
\hline$\geq 200$ & 8 & 2 & & 1 & 8 & \\
\hline Tumour size $(\mathrm{cm})$ & & & & & & 0.135 \\
\hline$\leq 3$ & 10 & 8 & 0.596 & 0 & 18 & \\
\hline$>3$ & 8 & 3 & & 2 & 9 & \\
\hline Lobe & & & 0.036 & & & 0.377 \\
\hline Upper/middle & 1 & 5 & & 1 & 5 & \\
\hline Lower & 17 & 6 & & 1 & 22 & \\
\hline Stage & & & 0.45 & & & 0.532 \\
\hline I-II & 11 & 9 & & 1 & 19 & \\
\hline III-IV & 7 & 2 & & 1 & 8 & \\
\hline Histological subtype & & & 0.035 & & & 0.483 \\
\hline AIS and MIA & 2 & 6 & & 1 & 7 & \\
\hline IMA & 16 & 5 & & 1 & 20 & \\
\hline Pleural invasion & & & 0.369 & & & 0.089 \\
\hline Positive & 4 & 5 & & 2 & 7 & \\
\hline Negative & 14 & 6 & & 0 & 20 & \\
\hline Lymphatic permeation & & & 0.298 & & & 0.111 \\
\hline Positive & 8 & 2 & & 2 & 8 & \\
\hline Negative & 10 & 9 & & 0 & 19 & \\
\hline
\end{tabular}

AIS, adenocarcinoma in situ; MIA, minimally invasive adenocarcinoma; IMA, invasive mucinous adenocarcinoma.

patients with PPMA were not significantly associated with age, sex, smoking index, tumour size, stage, pleural invasion and lymphatic permeation. In addition, it was identified that KRAS mutations were more often observed in invasive mucinous adenocarcinomas compared with AIS or MIA, and additionally more frequently observed in tumours that were localised to the lower lobe than in the upper or middle lobe of the lung.

Mucinous differentiation in pulmonary adenocarcinoma appears to occur early during carcinogenesis, and is most likely already present in the precursor lesion (21). Survival data for patients with PPMA have been limited due to its low incidence and the results of the small number of published studies have been conflicting (26). Shim et al (26) demonstrated that there was no significant difference in recurrence-free survival between patients with invasive mucinous adenocarcinoma and other patients. In the present study, all incidences of recurrence were limited to the lungs and extrapulmonary metastases were not detected in the pulmonary mucinous adenocarcinoma cohort, even though patients with invasive mucinous adenocarcinoma exhibited a tendency towards an improved recurrence-free survival.

In conclusion, primary pulmonary mucinous adenocarcinoma is a rare type of low grade malignant tumour. KRAS mutations may promote lineage-specific tumorigenesis in a pathological subtype of lung adenocarcinoma. The most effective treatment for PPMA is surgical resection, which demonstrated a tendency towards improved survival. The results of the present study may be crucial for the development of effective treatment for patients with PPMA. Additional validation studies are required to improve the targeted therapy strategies for mucinous adenocarcinoma. 


\section{References}

1. Qu Y, Che N, Zhao D, Zhang C, Su D, Zhou L, Zhang L, Wang C, Zhang $\mathrm{H}$ and Wei L: The clinicopathological significance of ALK rearrangements and KRAS and EGFR mutations in primary pulmonary mucinous adenocarcinoma. Tumour Biol 36 6417-6424, 2015

2. Cai D, Li H, Wang R, Li Y, Pan Y, Hu H, Zhang Y, Gong R, Pan B, Sun Y and Chen H: Comparison of clinical features, molecular alterations and prognosis in morphological subgroups of lung invasive mucinous adenocarcinoma. Onco Targets Ther 7: 2127-2132, 2014.

3. Sambrook-Gowar FJ: An unusual mucous cyst of the lung Thorax 33: 796-799, 1978.

4. Kragel PJ, Devaney KO, Meth BM, Linnoila I, Frierson HF Jr and Travis WD: Mucinous cystadenoma of the lung. A report of two cases with immunohistochemical and ultrastructural analysis. Arch Pathol Lab Med 114: 1053-1056, 1990.

5. Gaikwad A, Gupta A, Hare S, Gomes M, Sekhon H, Souza C, Inacio J, Lad S and Seely J: Primary adenocarcinoma of lung: A pictorial review of recent updates. Eur J Radiol 81: 4146-4155, 2012.

6. Travis WD, Brambilla E, Nicholson AG, Yatabe Y, Austin JHM Beasley MB, Chirieac LR, Dacic S, Duhig E, Flieder DB, et al: The 2015 world health organization classification of lung tumors: Impact of genetic, clinical and radiologic advances since the 2004 classification. J Thorac Oncol 10: 1243-1260, 2015.

7. Casali C, Rossi G, Marchioni A, Sartori G, Maselli F, Longo L, Tallarico E and Morandi U: A single institution-based retrospective study of surgically treated bronchioloalveolar adenocarcinoma of the lung: Clinicopathologic analysis, molecular features and possible pitfalls in routine practice. J Thorac Oncol 5: 830-836, 2010

8. Zhang Y, Sun Y, Xiang J, Zhang Y, Hu H and Chen H: A clinicopathologic prediction model for postoperative recurrence in stage Ia non-small cell lung cancer. J Thorac Cardiovasc Surg 148: 1193-1199, 2014

9. Hwang DH, Sholl LM, Rojas-Rudilla V, Hall DL, Shivdasani P, Garcia EP, MacConaill LE, Vivero M, Hornick JL, Kuo FC, et al: KRAS and NKX2-1 mutations in invasive mucinous adenocarcinoma of the lung. J Thorac Oncol 11: 496-503, 2016.

10. Rao G, Pierobon M, Kim IK, Hsu WH, Deng J, Moon YW, Petricoin EF, Zhang YW, Wang Y and Giaccone G: Inhibition of AKT1 signaling promotes invasion and metastasis of non-small cell lung cancer cells with K-RAS or EGFR mutations. Sci Rep 7: $7066,2017$.

11. Minamimoto R, Jamali M, Gevaert O, Echegaray S, Khuong A, Hoang CD, Shrager JB, Plevritis SK, Rubin DL, Leung AN, et al: Prediction of EGFR and KRAS mutation in non-small cell lung cancer using quantitative 18F FDG-PET/CT metrics. Oncotarget 8: 52792-52801, 2017.

12. Kamata T, Yoshida A, Shiraishi K, Furuta K, Kosuge T, Watanabe S, Asamura H and Tsuta K: Mucinous micropapillary pattern in lung adenocarcinomas: A unique histology with genetic correlates. Histopathology 68: 356-366, 2016.

13. Ichinokawa H, Ishii G, Nagai K, Kawase A, Yoshida J, Nishimura M, Hishida T, Ogasawara N, Tsuchihara K and Ochiai A: Distinct clinicopathologic characteristics of lung mucinous adenocarcinoma with KRAS mutation. Hum Pathol 44: 2636-2642, 2013.

14. Travis WD, Brambilla E, Noguchi M, Nicholson AG, Geisinger KR, Yatabe Y, Beer DG, Powell CA, Riely GJ, Van-Schil PE, et al: International association for the study of lung cancer/american thoracic society/european respiratory society international multidisciplinary classification of lung adenocarcinoma. J Thorac Oncol 6: 244-285, 2011.

15. Sakuma Y, Matsukuma S, Yoshihara M, Nakamura Y, Noda K, Nakayama H, Kameda Y, Tsuchiya E and Miyagi Y: Distinctive evaluation of nonmucinous and mucinous subtypes of bronchioloalveolar carcinomas in EGFR and K-ras gene-mutation analyses for Japanese lung adenocarcinomas: Confirmation of the correlations with histologic subtypes and gene mutations. Am J Clin Pathol 128: 100-108, 2007.
16. Yoshizawa A, Motoi N, Riely GJ, Sima CS, Gerald WL, Kris MG, Park BJ, Rusch VW and Travis WD: Impact of proposed IASLC/ATS/ERS classification of lung adenocarcinoma: prognostic subgroups and implications for further revision of staging based on analysis of 514 stage I cases. Mod Pathol 24: 653-664, 2011.

17. Warth A, Muley T, Meister M, Stenzinger A, Thomas M, Schirmacher P, Schnabel PA, Budczies J, Hoffmann H and Weichert W: The novel histologic International association for the study of lung Cancer/American thoracic society/European respiratory society classification system of lung adenocarcinoma is a stage-independent predictor of survival. J Clin Oncol 30: 1438-1446, 2012

18. Tsuta K, Kawago M, Inoue E, Yoshida A, Takahashi F, Sakurai H, Watanabe S, Takeuchi M, Furuta K, Asamura $\mathrm{H}$ and Tsuda $\mathrm{H}$ : The utility of the proposed IASLC/ATS/ERS lung adenocarcinoma subtypes for disease prognosis and correlation of driver gene alterations. Lung Cancer 81: 371-376, 2013.

19. Cadranel J, Quoix E, Baudrin L, Mourlanette P, Moro-Sibilot D, Morere JF, Souquet PJ, Soria JC, Morin F and Milleron B; IFCT-0401 Trial Group: IFCT-0401 Trial: A phase II study of gefitinib administered as first-line treatment in advanced adenocarcinoma with bronchioloalveolar carcinoma subtype. J Thorac Oncol 4: 1126-1135, 2009.

20. Righi L, Vatrano S, Di-Nicolantonio F, Massa F, Rossi G, Cavazza A, Volante M, Votta A, Izzo S, Lo-Iacono M, et al: Retrospective multicenter study investigating the role of targeted next-generation sequencing of selected cancer genes in mucinous adenocarcinoma of the lung. J Thorac Oncol 11: 504-515, 2016.

21. Geles A, Gruber-Moesenbacher U, ManzlC F, Al Effah M, Grygar E, Juettner-Smolle F and Popper HH: Pulmonary mucinous adenocarcinomas: Architectural patterns in correlation with genetic changes, prognosis and survival. Virchows Arch 467: 675-686, 2015 .

22. KuniiR,Jiang S,Hasegawa G, Yamamoto T,Umezu H, Watanabe T, Tsuchida M, Hashimoto T, Hamakubo T, Kodama T, et al: The predominant expression of hepatocyte nuclear factor $4 \alpha(\mathrm{HNF} 4 \alpha)$ in thyroid transcription factor-1 (TTF-1)-negative pulmonary adenocarcinoma. Histopathology 58: 467-476, 2011.

23. Finberg KE, Sequist LV, Joshi VA, Muzikansky A, Miller JM, Han M, Beheshti J, Chirieac LR, Mark EJ and Iafrate AJ: Mucinous differentiation correlates with absence of EGFR mutation and presence of KRAS mutation in lung adenocarcinomas with bronchioloalveolar features. J Mol Diagn 9: 320-326, 2007.

24. Watanabe H, Saito H, Yokose T, Sakuma Y, Murakami S, Kondo T, Oshita F, Ito $H$, Nakayama H, Yamada $K$ and Iwazaki M: Relation between thin-section computed tomography and clinical findings of mucinous adenocarcinoma. Ann Thorac Surg 99: 975-981, 2015.

25. Hata A, Katakami N, Fujita S, Kaji R, Imai Y, Takahashi Y, Nishimura T, Tomii K and Ishihara K: Frequency of EGFR and KRAS mutations in Japanese patients with lung adenocarcinoma with features of the mucinous subtype of bronchioloalveolar carcinoma. J Thorac Oncol 5: 1197-1200, 2010.

26. Shim HS, Kenudson M, Zheng Z, Liebers M, Cha YJ, Hoang Ho Q, Onozato M, Phi Le L, Heist RS and Iafrate AJ: Unique Genetic and survival characteristics of invasive mucinous adenocarcinoma of the lung. J Thorac Oncol 10: 1156-1162, 2015. 